\title{
Orally administered emu oil decreases acute inflammation and alters selected small intestinal parameters in a rat model of mucositis
}

\author{
Ruth J. Lindsay ${ }^{1}$, Mark S. Geier ${ }^{2}$, Roger Yazbeck ${ }^{3,4}$, Ross N. Butler ${ }^{5}$ and Gordon S. Howarth ${ }^{1,4 *}$ \\ ${ }^{1}$ School of Animal and Veterinary Sciences, Roseworthy Campus, University of Adelaide, Roseworthy, SA, Australia \\ ${ }^{2}$ South Australian Research and Development Institute, Pig and Poultry Production Institute, Nutrition Research Laboratory, \\ Roseworthy, SA, Australia \\ ${ }^{3}$ School of Biological Sciences, Flinders University, Adelaide, SA, Australia \\ ${ }^{4}$ Centre for Paediatric and Adolescent Gastroenterology, Children, Youth and Women's Health Service, North Adelaide, \\ SA, Australia \\ ${ }^{5}$ Sansom Institute, University of South Australia, Adelaide, SA, Australia
}

(Received 11 September 2009 - Revised 11 February 2010 - Accepted 12 February 2010 - First published online 9 April 2010)

\begin{abstract}
Mucositis resulting from cancer chemotherapy is a serious disorder of the alimentary tract. Emu oil has demonstrated anti-inflammatory properties in animal models of arthritis and wound healing; however, its effects on the intestine remain unknown. We investigated emu oil for its potential to decrease the severity of mucositis in a rat model. Female Dark Agouti rats $(110-150 \mathrm{~g})$ were orogastrically gavaged with emu oil $(0 \cdot 5$ or $1 \mathrm{ml})$ or water $(1 \mathrm{ml})$ for $5 \mathrm{~d}$ before intraperitoneal injection of 5 -fluorouracil $(5-\mathrm{FU}, 150 \mathrm{mg} / \mathrm{kg}$ ) or saline (control), and this was continued up to the day of sacrifice (48, 72 and $96 \mathrm{~h}$ post 5-FU administration). Histological (villus height, crypt depth (CD) and disease severity score) and biochemical (myeloperoxidase (MPO) activity) parameters were determined in intestinal tissues collected at sacrifice. Sucrase activity in vivo was quantified by the sucrose breath test. Activated neutrophil activity (MPO) in the ileum was significantly decreased by emu oil (0.5 ml, 451 (SEM 168$)$ U/g and $1 \mathrm{ml}, 503$ (SEM 213) U/g) compared with 5-FU-treated controls (1724 (SEM 431) U/g) 96 h post 5-FU administration. There were also significant increases in $\mathrm{CD}(152(\mathrm{SEM} 8) \mu \mathrm{m})$ in the ileum of rats that receivied $1 \mathrm{ml}$ emu oil at $96 \mathrm{~h}$ compared with 5-FU-treated controls $(\mathrm{CD}$ (106 $($ SEM 12) $\mu \mathrm{m})$ ). Emu oil did not affect sucrase activity. Emu oil decreased acute ileal inflammation, and improved mucosal architecture in the intestine during recovery from chemotherapy in rats. Further studies investigating the potential benefits of emu oil as a nutritional supplement for the treatment of intestinal disorders are indicated.
\end{abstract}

Rat model: Emu oil: Mucositis: 5-Fluorouracil: Chemotherapy

Mucositis is a common side-effect of cancer chemotherapy ${ }^{(1)}$, which drastically reduces the quality of life of patients undergoing treatment. Mucositis is a debilitating condition that can occur in all the regions of the gastrointestinal tract ${ }^{(1,2)}$; however, it most commonly affects the mucosa of the mouth (oral mucositis) and small intestine (intestinal mucositis). It is characterised by erosion and deterioration of the mucosa with symptoms including severe pain and bloating, diarrhoea and nausea ${ }^{(2,3)}$. Chemotherapy-induced enterocyte apoptosis can ultimately result in the failure of gut barrier function, allowing translocation of pathogenic bacteria and endotoxins $^{(4)}$ into the bloodstream, resulting in systemic infection $^{(3)}$. The rate of infection and sepsis in mucositis patients is directly proportional to the severity of the condition $^{(3)}$. Mucositis remains one of the primary determinants of morbidity and mortality in patients undergoing treatment for cancer ${ }^{(5)}$ as a consequence of these secondary complications. Indeed, symptoms can progress to the stage whereby chemotherapy must be ceased ${ }^{(3)}$. Currently, there are no effective treatments for intestinal mucositis ${ }^{(6,7)}$, although a number of agents are undergoing investigation, including keratinocyte growth factor- $1^{(2,8,9)}$, insulin-like growth factor- $\mathrm{I}^{(10,11)}$, whey growth factor extract ${ }^{(12,13)}$ and velafermin (fibroblast growth factor-20) ${ }^{(14)}$.

The emu (Dromaius novaehollandiae) is a large flightless bird of the order Struthioniformes, traditionally endemic to Australia, but now farmed around the world for its meat, leather and, most recently, oil ${ }^{(15)}$. Emu oil is extracted from both the subcutaneous and retroperitoneal fat of the bird by first rendering the macerated tissue, and then passing the liquefied fat through a series of filters to extract a purified oil $^{(16)}$. Some manufacturers also use centrifugation to separate the oil from other extraneous components of the adipose tissue. Emu oil is predominantly composed of fatty acids (FA), with a lipid content of $98.8 \%$ for the subcutaneous adipose tissue and $98.0 \%$ for the retroperitoneal adipose tissue $^{(16)}$. Oleic acid constitutes approximately $43-46 \%$ of the FA component, with linoleic acid $(9.6 \%)$, palmitic acid

Abbreviations: 5-FU, 5-fluorouracil; CD, crypt depth; FA, fatty acids; JI, jejunum-ileum; MPO, myeloperoxidase; VH, villus height

* Corresponding author: Gordon S. Howarth, fax +61 88303 7972, email gordon.howarth@adelaide.edu.au 
$(23.5 \%)$, stearic acid (9.1\%) and linolenic acid (0.6\%) also being present. The composition of the remaining $1-2 \%$ is yet to be conclusively defined, although natural antioxidants, such as carotenoids and flavones, and skin permeation-enhancing factors have been identified in oil preparations ${ }^{(15)}$.

Emu oil was first used by indigenous Australians, and then by early European settlers, to provide relief from pain and to promote wound healing ${ }^{(15)}$. Emu oil has also been reported to possess analgesic properties ${ }^{(15)}$; however, there have been no rigorous experimental studies to support these claims. Recent studies have focussed on the effects of emu oil on arthritis and dermal inflammation in animal models ${ }^{(15,17-19)}$. Topical application of emu oil to animals has been shown to reduce the levels of tumour necrosis factor- $\alpha$ and other proinflammatory cytokines in a model of adjuvant-induced inflammation $^{(17)}$. These cytokines are known to be involved in the pathogenesis of mucositis, thus indicating a potential role for emu oil in the treatment of chemotherapy-induced intestinal inflammation ${ }^{(15,17,18)}$

The promising anti-inflammatory effects displayed by emu oil in previous studies ${ }^{(15,17,18,20)}$ suggest the potential for therapeutic benefit in chemotherapy-induced mucositis. Accordingly, we hypothesised that emu oil ingestion would decrease the severity of intestinal damage induced by the chemotherapeutic drug, 5-fluorouracil (5-FU), in a rat model of mucositis, potentially through the inflammation-modulating effects of the $n-3$ and $n-9$ FA constituents of the oil.

\section{Materials and methods \\ Animal studies}

Female Dark Agouti rats ( $n$ 80; 110-150g) were housed in individual metabolic cages (Tecniplast, Exton, PA, USA) in a climate- and light-controlled room, and were given ad libitum access to water and food $(18 \%$ casein-based $\left.\operatorname{diet}^{(21)}\right)$. Rats were randomly allocated to one of the following groups ( $n$ 8): 5-FU (Mayne Pharma Pty Ltd, Mulgrave, Vic, Australia) and water, 48, 72 and $96 \mathrm{~h}, 5-\mathrm{FU}$ and $0.5 \mathrm{ml}$ emu oil, 48, 72 and $96 \mathrm{~h}$; and 5-FU and $1 \mathrm{ml}$ emu oil, 48, 72 and $96 \mathrm{~h}$. A negative control group was gavaged with water, and it received a saline injection instead of 5-FU. Emu oil was prepared by utilising specific methodologies developed for Technology Investment Corporation by Emu Tracks Pty Ltd (Marleston, Adelaide, SA, Australia). Briefly, these processes involved the rendering and filtration of emu adipose tissue, with appropriate considerations for delivery of quality assurance and product consistency. The complete FA composition of the emu oil utilised in the present study is listed in Table 1. All animal experiments adhered to the Australian Code of Practice for the Care and Use of Animals for Scientific Purposes, and were approved by the Animal Ethics Committees of the University of Adelaide and the Children, Youth and Women's Health Service.

Emu oil or water was administered daily via orogastric gavage between -120 and $72 \mathrm{~h}$ of the experimental period (Fig. 1). At $0 \mathrm{~h}$, all rats were intraperitoneally injected with a single dose of either saline (control group) or 5-FU $(150 \mathrm{mg} / \mathrm{kg}$; Fig. 1). Body weight, feed/water intake and faecal/urine output were monitored and recorded on a daily
Table 1. Complete fatty acid analysis of the emu oil used in the present study

\begin{tabular}{lc}
\hline Fatty acid & Total $(\%)$ \\
\hline $16: 0$ & $25 \cdot 80$ \\
$16: 1 n-7$ & $4 \cdot 08$ \\
$16: 1 n-9$ & $0 \cdot 12$ \\
$16: 2 n-3$ & $0 \cdot 04$ \\
$17: 1$ & $0 \cdot 06$ \\
$18: 0$ & $10 \cdot 17$ \\
$18: 1 n-7$ & $2 \cdot 19$ \\
$18: 1 n-9$ & $48 \cdot 02$ \\
$18: 1 n-12$ & $0 \cdot 02$ \\
$18: 2 n-6$ & $7 \cdot 74$ \\
$18: 2 n-9$ & $0 \cdot 04$ \\
$18: 3 n-3$ & $0 \cdot 69$ \\
trans-18:1n-9 & $0 \cdot 29$ \\
trans-18:2 & $0 \cdot 12$ \\
$20: 0$ & $0 \cdot 11$ \\
$20: 1 n-9$ & $0 \cdot 33$ \\
$20: 2 n-6$ & 0.06 \\
$20: 3 n-3$ & $0 \cdot 04$ \\
$20: 3 n-9$ & $0 \cdot 03$ \\
$22: 5 n-3$ & $0 \cdot 02$ \\
$22: 6 n-3$ & 0.03 \\
$24: 0$ & $0 \cdot 02$ \\
\hline
\end{tabular}

basis. Rats were killed 48, 72 or $96 \mathrm{~h}$ post 5 -FU injection (Fig. 1) by $\mathrm{CO}_{2}$ asphyxiation followed by cervical dislocation.

\section{Sucrose breath test}

The sucrose breath test was performed as a non-invasive assessment of small intestinal brush border disaccharidase activity $^{(22)}$. The sucrose breath test was performed before the commencement of the trial $(-120 \mathrm{~h})$, immediately before 5-FU injection $(0 \mathrm{~h})$ and before killing $(48,72,96 \mathrm{~h}$ post injection; Fig. 1). After an overnight fast, rats were gavaged with $1 \mathrm{ml}$ of $0.25 \mathrm{~g} / \mathrm{ml}$ sucrose solution, naturally labelled with ${ }^{13} \mathrm{C}$. Rats were then placed in sealed plastic containers for $2 \mathrm{~min}$, before a $10 \mathrm{ml}$ sample of breath was collected into an evacuated tube. Breath samples were collected at 0,15 , $30,45,60,75,90,105$ and $120 \mathrm{~min}$ after the administration of the sucrose solution. Breath samples were analysed for ${ }^{13} \mathrm{C}$ content using isotope ratio mass spectrometer. Data were expressed as mean percentage cumulative dose at $90 \mathrm{~min}$ after sucrose gavage as described previously ${ }^{(22)}$. Percentage cumulative dose at $90 \mathrm{~min}$ is the percentage of ${ }^{13} \mathrm{C} \mathrm{dose} / \mathrm{h}$ recovered in the breath $90 \mathrm{~min}$ post sucrose administration. This gives an indirect indication of the rate at which sucrose is being cleaved by sucrase in the small intestine, and therefore, how much sucrase is present on the enterocytes in the small intestine. The sucrose breath test has been found to correlate significantly with in vitro sucrase activity $(r 0 \cdot 85)^{(23)}$.

\section{Tissue collection}

After killing by $\mathrm{CO}_{2}$ asphyxiation and cervical dislocation, the gastrointestinal tract of each animal was removed, measured, emptied of contents and weighed. Segments $(2 \mathrm{~cm})$ were removed from the duodenum, jejunum, jejunum-ileum (JI) junction, ileum and colon, and were placed in $10 \%$ buffered formalin for histological analysis. In addition, segments 


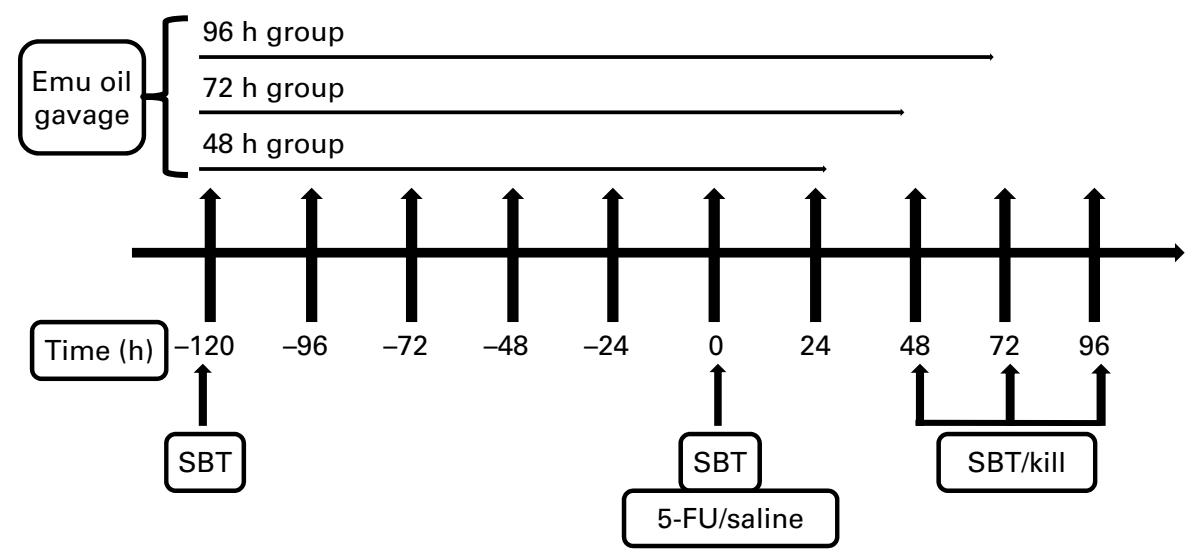

Fig. 1. Time line of the animal trial summarising gavage, sucrose breath test, saline or 5 -fluorouracil (5-FU) injection and kill events. SBT, sucrose breath test.

$(4 \mathrm{~cm})$ directly adjacent to the corresponding histological samples were collected and snap-frozen in liquid $\mathrm{N}_{2}$ for biochemical analyses. Samples were stored at $-80^{\circ} \mathrm{C}$, until prepared for analysis by homogenisation in $10 \mathrm{~mm}$ phosphate buffer. The remaining visceral organs (thymus, spleen, lungs, heart, liver and kidneys) were weighed and discarded.

\section{Biochemical analysis}

Myeloperoxidase (MPO) levels in the small intestine were determined as an indicator of neutrophil infiltration, and hence, acute inflammation, using techniques described by Howarth et al. ${ }^{(12)}$. Thawed, homogenised samples were centrifuged at $13000 \mathrm{~g}$ for $10 \mathrm{~min}$, after which the supernatant was discarded, and the tissue homogenate was re-suspended in $0.5 \%$ hexadecyltrimethyl ammonium bromide. After vortexing for $2 \mathrm{~min}$, samples were again centrifuged $(5000 \mathrm{~g}$ for $2 \mathrm{~min}$ ). The supernatants were then aliquoted into ninetysix-well plates. After the addition of an $o$-dianisidine reagent, the change in absorbance was measured at $450 \mathrm{~nm}$ (Sunrise Microplate Absorbance Reader, Tecan Austria $\mathrm{GmbH}$, Grödig, Austria).

\section{Histological analyses}

Samples of small intestine were transferred from $10 \%$ buffered formalin into $70 \%$ ethanol $24 \mathrm{~h}$ after collection. Specimens were then routinely processed and embedded in paraffin wax, and $4 \mu \mathrm{m}$ sections were prepared and stained with haemotoxylin and eosin. Small intestinal crypt depth (CD) and villus height (VH) were measured in the jejunum, JI junction and ileum (forty villi and forty crypts per section $)^{(12)}$. Overall histological damage severity of intestinal sections was also assessed and scored semi-quantitatively according to methods described by Howarth et al. ${ }^{(12)}$. The parameters assessed were villus/crypt ratio, enterocyte disruption, reduction in goblet cell numbers, crypt disruption, crypt cell disruption, lymphocyte and polymorphonucleocyte infiltration, thickening/oedema of the submucosa and thickening of the muscularis externa ${ }^{(12)}$. All analyses were performed in a blinded fashion using a light microscope (Olympus BH-2, Tokyo, Japan), digital camera (Sony, Tokyo, Japan) and Image Pro-Plus Software Package version 4.5.1.2.7 (Media Cybernetics, Silver Spring, MD, USA).

\section{Statistical analysis}

Statistical analyses were conducted using SPSS 15.0.1 for Windows (SPSS, Inc., Chicago, IL, USA). Daily metabolic data were analysed using a repeated measures ANOVA with Holme's post hoc test $(P<0.05$ significance $)$ to compare the differences both among groups and within groups across the duration of the trial. Overall histological damage severity was compared by a Kruskal-Wallis test with Bonferroni's post hoc test, and was expressed as median (range). All other data were compared by one-way ANOVA with Tukey's post hoc test, and were expressed as means with their standard errors. For all the data, $P<0.05$ was considered significant.

\section{Results}

Before the administration of 5-FU, all rats gained body weight, with no significant differences among groups ( $P>0.05$; Fig. 2). Following 5-FU/saline administration, all the 5-FU-injected rats, including those treated with emu oil, experienced significant weight loss $(P<0 \cdot 05)$. This decrease in body weight continued over the remainder of the

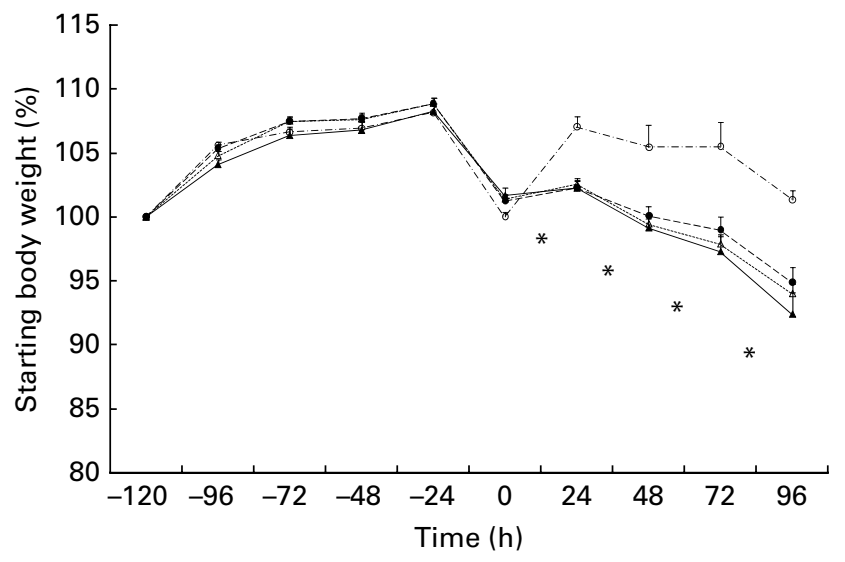

Fig. 2. Body weight change from -120 to $96 \mathrm{~h}$ in rats gavaged with water, $0.5 \mathrm{ml}$ emu oil (EO) or $1 \mathrm{ml} \mathrm{EO}$, and intraperitoneally injected with saline or 5-fluorouracil (5-FU) at $0 \mathrm{~h}$. Data are expressed as starting body weight $(\%)$ means with their standard errors. ${ }^{*}$ Mean values were significantly different when compared with saline for all the 5-FU-treated groups $(P<0.05)$. $-\ominus \cdot-$, Saline/water; -๑-., 5-FU/water; ---A---, 5-FU/0.5 ml EO; ——, $5-\mathrm{FU} / 1 \mathrm{ml}$ EO. 


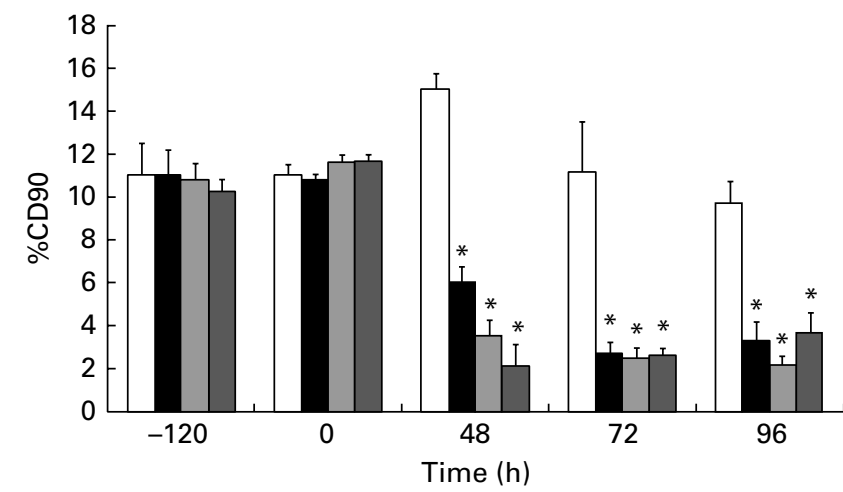

Fig. 3. Small intestinal sucrase activity in rats gavaged with water, 0.5 or $1 \mathrm{ml}$ emu oil (EO), and intraperitoneally injected with saline or 5 -fluorouracil $(5-\mathrm{FU})$ at $\mathrm{Oh}$, which was measured using the ${ }^{13} \mathrm{C}$-sucrose breath test at time points $-120,0,48,72$ and $96 \mathrm{~h}$. Data are expressed as (percentage cumulative dose at $90 \mathrm{~min}$ ) means with their standard errors. * Mean values were significantly different when compared with saline $(P<0.05)$. $\square$, Saline/ water; $\mathbf{\square}, 5-\mathrm{FU} /$ water; $\square, 5-\mathrm{FU} / 0.5 \mathrm{ml} \mathrm{EO}$; $\square, 5-\mathrm{FU} / 1 \mathrm{ml} \mathrm{EO}$.

experimental period, with no significant differences between the 5-FU groups (Fig. 2). Normal animals maintained weight for the duration of the trial.

Brush border sucrase activity was used as an indicator of the health and maturity of the epithelial cells which line the intestine. No significant differences in percentage cumulative dose at $90 \mathrm{~min}$ were observed at $120 \mathrm{~h}$ before and immediately before 5-FU/saline administration (Fig. 3). However, at 48, 72 and $96 \mathrm{~h}$ post $5-\mathrm{FU} / \mathrm{saline}$ administration, a significant decrease of up to $87 \%$ in percentage cumulative dose at 90 min was evident in all the rats that received 5-FU, including those treated with emu oil, with no significant differences being observed among rats in all the 5-FU-treated groups. Normal rats maintained percentage cumulative dose at $90 \mathrm{~min}$ at initial levels for the duration of the trial.

Administration of 5-FU induced a significant increase $(275 \%)$ in MPO activity in the jejunum relative to normal controls $(P<0.0001$; Fig. 4(a)). One millilitre of emu oil administered to 5-FU-treated animals significantly decreased MPO activity compared with 5-FU controls at 48 and $96 \mathrm{~h}$ (Fig. 4(a)). The lower dose of emu oil $(0.5 \mathrm{ml})$ exerted a lesser effect, with no statistically significant difference between $0.5 \mathrm{ml}$ emu oil-injected groups and either the normal or 5-FU-injected controls at $48 \mathrm{~h}$ (Fig. 4(a)). At $72 \mathrm{~h}$, 5 -FU significantly elevated the levels of MPO in the jejunum in all the groups compared with normal controls $(P<0 \cdot 0001)$. At both the doses, emu oil had no effect on MPO activity at this time point $(\mathrm{P}>0.05$; Fig. 4(a)).

In the mid-small intestine (JI), 5-FU did not induce a significant elevation in MPO levels at 48 and $72 \mathrm{~h}$ $(P<0 \cdot 05)$. Neither dose of emu oil altered MPO levels within the JI relative to the normal or 5-FU controls at these early time points $(P>0.05)$. At $96 \mathrm{~h}$, however, both the 0.5 and $1 \mathrm{ml}$ emu oil doses significantly reduced the levels of MPO compared with 5-FU controls $(P<0 \cdot 05$; Fig. 4(b)).

In the ileum, no significant reduction in MPO levels with either dose of emu oil occurred at the early 48 and $72 \mathrm{~h}$ time points relative to 5 -FU controls $(P>0.05)$; however, at $96 \mathrm{~h}, \mathrm{MPO}$ activity was decreased to levels comparable to that of normal rats $(P<0.01$; Fig. $4(\mathrm{c}))$.
Small intestinal sections stained with haemotoxylin and eosin were examined for 5-FU-induced damage, which manifests as shortened villi and crypts, due to reduced cellularity ${ }^{(6)}$. In the recovery phase, it is usual for $\mathrm{CD}$ to increase above normal, as cell proliferation is stimulated to repair damage sustained from 5-FU administration ${ }^{(24)}$. At $48 \mathrm{~h}$ post 5 -FU administration, emu oil $(1 \mathrm{ml})$ only minimally improved $\mathrm{VH}$ in the proximal intestine compared with 5-FU-treated control rats, although this was not maintained in the more distal
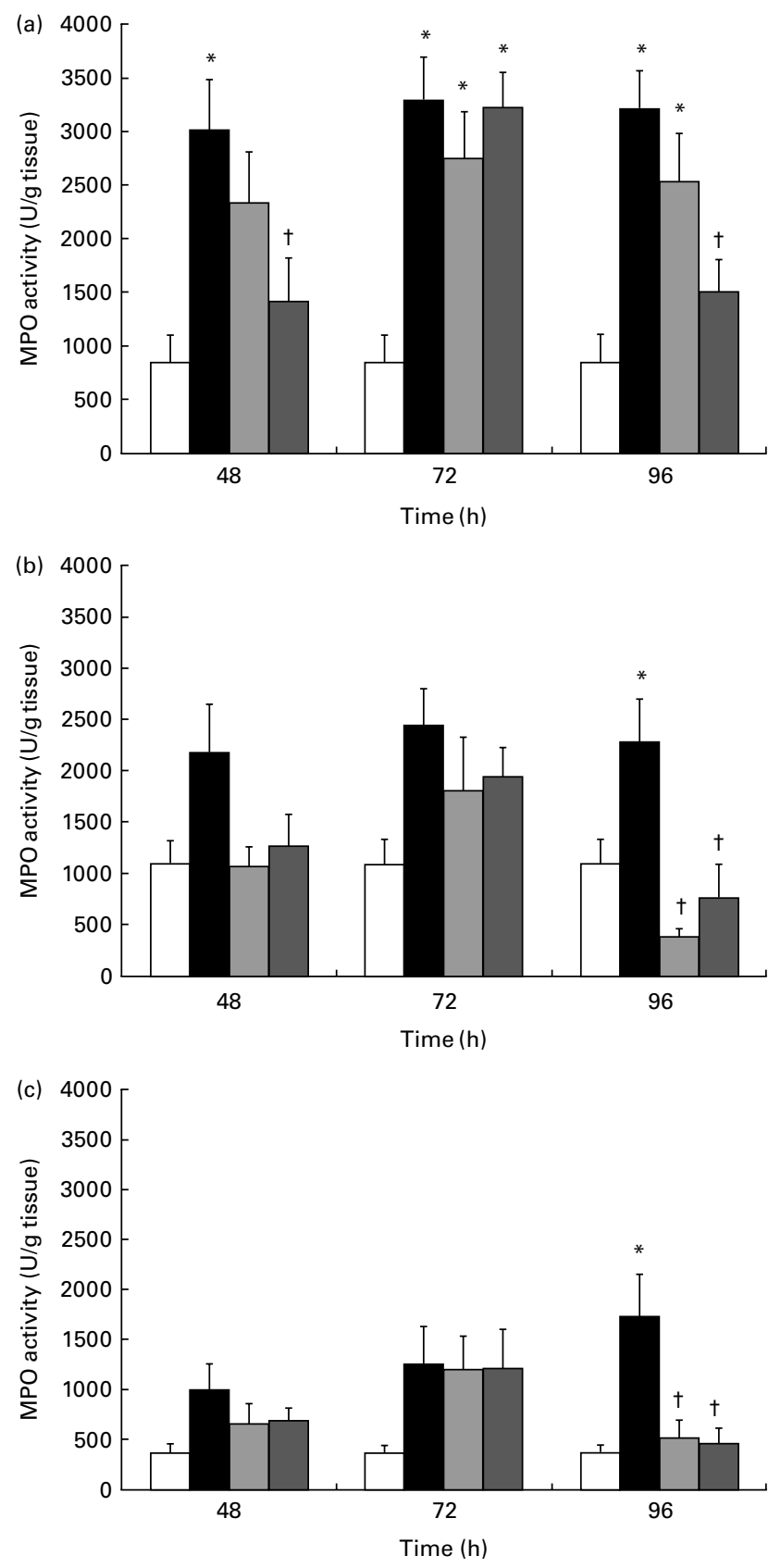

Fig. 4. Myeloperoxidase (MPO) activity in the jejunum (a), jejunum-ileum junction (b) and ileum (c) at 48, 72 and $96 \mathrm{~h}$. Data are expressed as (MPO units/g tissue) means with their standard errors. *Mean values were significantly different when compared with saline $(P<0.05)$. † Mean values were significantly different when compared with 5 -fluorouracil $(5-\mathrm{FU})+$ water $(P<0.05)$. $\square$, Saline/water; $\mathbf{\square}, 5-\mathrm{FU} /$ water; $\square, 5-\mathrm{FU} / 0.5 \mathrm{ml}$ emu oil; $\square, 5-F U / 1 \mathrm{ml}$ emu oil. 


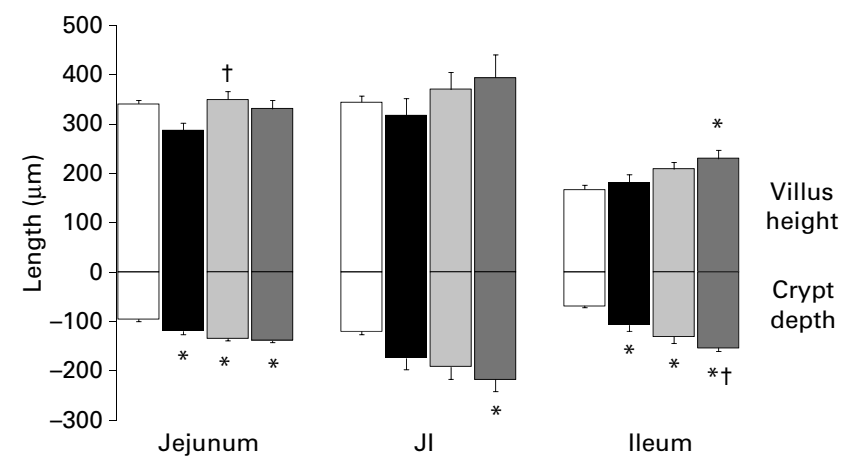

Fig. 5. Villus height and crypt depth in the jejunum, jejunum-ileum junction (JI) and ileum at $96 \mathrm{~h}$. Data are expressed as means with their standard errors. * Mean values were significantly different when compared with saline $(P<0.05)$. †Mean values were significantly different when compared with 5-fluorouracil 5-(FU) + water $(P<0.05)$. $\square$, Saline/water; $\mathbf{\square}, 5$-Fu/water; $\square, 5-\mathrm{Fu} / 0.5 \mathrm{ml}$ emu oil; $\square, 5-\mathrm{Fu} / 1 \mathrm{ml}$ emu oil.

regions of the intestine, the JI and ileum (data not shown). At $72 \mathrm{~h}$ post 5 -FU administration, no effect was apparent with either dose of emu oil in any section of the intestine, with no significant differences among all the 5-FU-treated groups $(P>0.05)$. In the jejunum $96 \mathrm{~h}$ post 5 -FU administration, only the lower dose of emu oil increased $\mathrm{VH}$ relative to 5-FU controls, with no other significant effects being observed. This was also evident in the JI, where emu oil had no apparent effect on VH $(P>0 \cdot 05)$. In the ileum, only the higher dose of emu oil $(1 \mathrm{ml})$ was able to significantly increase $\mathrm{VH}$ in relation to the normal control (Fig. 5). At 48 and $72 \mathrm{~h}$, there were no significant differences in CD among treatments $(P>0.05)$, with the exception of the jejunum at $48 \mathrm{~h}$, where 5 -FU significantly shortened crypts relative to normal rats. The greatest differences in $\mathrm{CD}$ were at $96 \mathrm{~h}$, when, in the jejunum and ileum, the crypts in all the 5-FU-treated rats were significantly longer compared with normal controls (Fig. 5). In the JI, only the high dose of emu oil was able to significantly lengthen crypts relative to the normal controls (Fig. 5).

5-FU injection induced a significant increase in the histological severity scores compared with normal rats (unpublished results). No significant differences in severity scores were observed among the 5-FU-treated groups at any time point $(P>0.05)$.

\section{Discussion}

In the present study, emu oil was unable to impact significantly on the induction phase of mucositis, suggesting that it was incapable of decreasing the peak severity of mucositis. However, although emu oil did not appear to exert any protective effects on the intestine, there were indications that the rate of recovery from mucositis could be improved. Emu oil altered specific parameters associated with 5-FU-induced damage in the recovery stage of mucositis $(96 \mathrm{~h})$, relative to the untreated controls, including a significant decrease in activated neutrophil infiltration and improvements in CD and VH. The emu oil-induced effects did not appear to be dose dependent. This proof-of-concept study represents the first report of decreased intestinal inflammation following oral administration of emu oil. The decrease in acute inflammation, as indicated by decreased MPO activity, is supported by previous studies in which topical application of emu oil reduced the severity of arthritis and dermal inflammation $^{(17-20)}$. A number of hypotheses relate to the potential mechanism by which emu oil exerts its anti-inflammatory effects. It has been suggested that the $n-3$ and $n-9$ FA present in emu oil may be involved in its anti-inflammatory action ${ }^{(17)}$. Yoganathan et al. ${ }^{(17)}$ proposed that the anti-inflammatory properties of emu oil are not fully explained by the FA profile, and that other components of emu oil, such as tocopherols, carotenoids and flavones, may exert additional anti-inflammatory $^{(25)}$ and anti-oxidant ${ }^{(26)}$ effects. A recent study by Bennet et $a l .{ }^{(27)}$ has suggested that the radical-scavenging properties of emu oil were most likely due to the minor constituents in the non-TAG fraction of the oil ${ }^{(27)}$, which, in combination with the FA component, offered greater protection against oxidation. These antioxidants may have impacted on levels of damaging reactive oxygen species which are generated in the first of five recognised stages of mucositis $(3,6,28,29)$. The initial stage occurs immediately after the introduction of cytotoxins into the system, and it is these reactive oxygen species which are responsible caused to damage to cells, tissues and blood vessels ${ }^{(29)}$. In addition, a series of downstream effects occur, including an increase in the levels of secondary messengers and transcription factors such as $\mathrm{NF}-\kappa \mathrm{B}^{(6)}$, with subsequent up-regulation of secondary messengers and proinflammatory cytokines. Indeed, a previous study investigating croton oil-induced inflammation demonstrated that topical application of emu oil was able to decrease expression of proinflammatory cytokines such as TNF- $\alpha$, IL-1 $\beta$ and IL-6 ${ }^{(6)}$.

In the present study, emu oil maintained villus structure along the length of the small intestine, and at $96 \mathrm{~h}, \mathrm{CD}$ was significantly increased in the ileum, suggesting that emu oil had stimulated mucosal growth in the recovery phase of mucositis. Possible mechanisms for this observation include an increase in cell proliferation, a decrease in apoptosis, cell hypertrophy or a combination of these factors. Previous studies have shown that the intestine has the ability to grow and adapt if one section is severely damaged ${ }^{(30)}$. This has been demonstrated in short bowel syndrome in which compensatory alterations include increased $\mathrm{CD}$ and $\mathrm{VH}$ as well as enterocyte proliferation ${ }^{(30)}$. Haxhija et al. $^{(31)}$ reported that the adaptive response in the small intestine varied depending on the area of damage. Damage occurring predominantly in the proximal small intestine resulted in the cells in the ileum becoming hypertrophic; however, in response to ileal damage, jejunal cells became hyperplastic ${ }^{(31)}$. In the present study, compensatory hypertrophy may have occurred in the ileum, as 5-FU preferentially damages the upper small intestine due to the higher cell turnover rate. This response could have explained the increase in CD observed in the ileum.

Damage to the brush border of the small intestine, such as that resulting from mucositis, (i.e. ulceration, and/or shortening and blunting of the villus) has an adverse impact on sucrase expression ${ }^{(22)}$; therefore, sucrase activity was used as an indirect indicator of intestinal integrity and maturity. sucrose breath test results in the present investigation indicated no demonstrable maintenance of sucrase activity by emu oil, although a similar effect had been described in a previous study in which the effect of another lipid-based 
extract, Lyprinol ${ }^{\mathrm{TM}}$, was examined in the 5-FU mucositis model $^{(32)}$. Lyprinol ${ }^{\text {TM }}$ failed to improve sucrase activity, despite improvements in other parameters including $\mathrm{CD}$ and cell proliferation ${ }^{(32)}$. Histological analysis in the present study indicated that villus structure in both emu oil treatment groups did not significantly differ from that in the saline controls at any time point. The greater $\mathrm{CD}$ in the ileum that is evident at $96 \mathrm{~h}$ following the administration of emu oil may have been due to a rapid increase in crypt cell proliferation, in order to repair damage to the intestine; however, these cells may not have had sufficient time to mature and begin actively synthesising sucrase before migration up the villus. An increase in enterocyte proliferation may therefore have been a mechanism for emu oil action in the present study. Future studies, building on the positive indications found in this efficacy study, would benefit from the examination of different emu oil doses, inter-batch variation, comparison to other oils with known anti-inflammatory properties and the effects on the healthy gastrointestinal tract. In addition, identification of the individual bioactive factors would facilitate more targeted development of emu oil preparations with greater potency, and lead to a greater understanding of the underlying mechanisms of the changes observed in the present study.

In conclusion, emu oil was not able to maintain body weight and sucrase activity or to decrease histological severity scores following chemotherapy. However, it was able to decrease chemotherapy-associated inflammation in the small intestine, and alter the mucosal architecture in the recovery phase of mucositis. The promising results from the present study indicate that a further investigation of emu oil as a nutritional supplement to promote healing of the damaged intestine, following the resolution of cancer treatment, is required.

\section{Acknowledgements}

The authors would like to thank Kerry Lymn for a substantial contribution towards planning and conducting the animal trials, and Esther Staunton and Betty Zacharakis from the Gastroenterology Department of the Children, Youth and Women's Health Service for analysis of breath samples by isotope ratio mass spectrometry. R. J. L. conducted all animal trials and data analyses and prepared the manuscript. M. S. G. contributed to the experimental design and assisted in the manuscript preparation. R. Y. assisted with the animal trials and all data analyses. R. N. B. assisted in the manuscript preparation. G. S. H. contributed to the experimental design, analysis of data and manuscript preparation. G. S. H. is supported by a Sally Birch Cancer Council Australia Research Fellowship. This project was funded in part by the University of Adelaide and Emu Tracks Pty Ltd, Marleston, South Australia. There are no other conflicts of interest or financial agreements in place.

\section{References}

1. Bowen JM, Gibson RJ, Cummins AG, et al. (2006) Intestinal mucositis: the role of the Bcl-2 family, p53 and caspases in chemotherapy-induced damage. Support Care Cancer 14, 713-731.

2. Gibson RJ, Bowen JM \& Keefe DM (2005) Palifermin reduces diarrhea and increases survival following irinotecan treatment in tumor-bearing DA rats. Int J Cancer 116, 464-470.
3. Lalla RV \& Peterson DE (2006) Treatment of mucositis, including new medications. Cancer J 12, 348-354.

4. Choi K, Lee SS, Oh SJ, et al. (2007) The effect of oral glutamine on 5-fluorouracil/leucovorin-induced mucositis/stomatitis assessed by intestinal permeability test. Clin Nutr 26, 57-62.

5. Keefe DM (2004) Gastrointestinal mucositis: a new biological model. Support Care Cancer 12, 6-9.

6. Logan RM, Stringer AM, Bowen JM, et al. (2007) The role of pro-inflammatory cytokines in cancer treatment-induced alimentary tract mucositis: pathobiology, animal models and cytotoxic drugs. Cancer Treat Rev 33, 448-460.

7. Keefe DM, Gibson RJ \& Hauer-Jensen M (2004) Gastrointestinal mucositis. Semin Oncol Nurs 20, 38-47.

8. Gibson RJ, Keefe DMK, Clarke JM, et al. (2002) The effect of keratinocyte growth factor on tumour growth and small intestinal mucositis after chemotherapy in the rat with breast cancer. Cancer Chemother Pharmacol 50, 53-58.

9. von Bultzingslowen I, Brennan MT, Spijkervet FK, et al. (2006) Growth factors and cytokines in the prevention and treatment of oral and gastrointestinal mucositis. Support Care Cancer 14, 519-527.

10. Cool JC, Dyer JL, Xian CJ, et al. (2005) Pre-treatment with insulin-like growth factor-I partially ameliorates 5-fluorouracilinduced intestinal mucositis in rats. Growth Horm IGF Res 15, $72-82$.

11. Howarth GS (2003) Insulin-like growth factor-I and the gastrointestinal system: therapeutic indications and safety implications. J Nutr 133, 2109-2112.

12. Howarth GS, Francis GL, Cool JC, et al. (1996) Milk growth factors enriched from cheese whey ameliorate intestinal damage by methotrexate when administered orally to rats. J Nutr 126, 2519-2530.

13. Clarke J, Butler RN, Howarth GS, et al. (2002) Exposure of oral mucosa to bioactive milk factors reduces severity of chemotherapy-induced mucositis in the hamster. Oral Oncol 38, 478-485.

14. Gibson RJ, Stringer AM, Bowen JM, et al. (2007) Velafermin improves gastrointestinal mucositis following irinotecan treatment in tumor-bearing DA rats. Cancer Biol Ther 6, 541-547.

15. Whitehouse MW, Turner AG, Davis CK, et al. (1998) Emu oil(s): a source of non-toxic transdermal anti-inflammatory agents in aboriginal medicine. Inflammopharmacology 6, 1-8.

16. Beckerbauer LM, Thiel-Cooper R, Ahn DU, et al. (2001) Influence of two dietary fats on the composition of emu oil and meat. Poult Sci 80, 187-194.

17. Yoganathan S, Nicolosi R, Wilson T, et al. (2003) Antagonism of croton oil inflammation by topical emu oil in CD-1 mice. Lipids 38, 603-607.

18. Lopez A, Sims DE, Ablett RF, et al. (1999) Effect of emu oil on auricular inflammation induced with croton oil in mice. Am J Vet Res 60, 1558-1561.

19. Snowden JM \& Whitehouse MW (1997) Anti-inflammatory activity of emu oils in rats. Inflammopharmacology 5, 127-132.

20. Qiu XW, Wang JH, Fang XW, et al. (2005) Anti-inflammatory activity and healing-promoting effects of topical application of emu oil on wound in scalded rats. Di Yi Jun Yi Da Xиe Xиe Bao 25, 407-410.

21. Tomas FM, Murray AJ \& Jones LM (1984) Modification of glucocorticoid-induced changes in myofibrillar protein turnover in rats by protein and energy deficiency as assessed by urinary excretion of Ntau-methylhistidine. Br J Nutr 51, 323-337.

22. Pelton NS, Tivey DR, Howarth GS, et al. (2004) A novel breath test for the non-invasive assessment of small intestinal mucosal injury following methotrexate administration in the rat. Scand $J$ Gastroenterol 39, 2.

23. Tooley KL, Howarth GS, Lymn KA, et al. (2009) Optimization of the non-invasive ${ }^{13} \mathrm{C}$-sucrose breath test in a rat model of 
methotrexate-induced mucositis. Cancer Chemother Pharmacol 65, 913-921.

24. Cheng H \& Bjerknes M (1982) Whole population cell kinetics of mouse duodenal, jejunal, ileal, and colonic epithelia as determined by radioautography and flow cytometry. Anat Rec. 203, 251-264.

25. Singh U \& Jialal I (2004) Anti-inflammatory effects of alphatocopherol. Ann N Y Acad Sci 1031, 195-203.

26. Sylvester PW (2007) Vitamin E and apoptosis. Vitam Horm 76, 329-356.

27. Bennett DC, Code WE, Godin DV, et al. (2008) Comparison of the antioxidant properties of emu oil with other avian oils. Anim Prod Sci 48, 1345-1350.

28. Sonis ST (2004) Pathobiology of mucositis. Semin Oncol Nurs 20, $11-15$.
29. Sonis ST, Elting LS, Keefe D, et al. (2004) Perspectives on cancer therapy-induced mucosal injury: pathogenesis, measurement, epidemiology, and consequences for patients. Cancer 100, 1995-2025.

30. Drozdowski L \& Thomson ABR (2006) Intestinal mucosal adaptation. World J Gastroenterol 12, 4614-4627.

31. Haxhija EQ, Yang H, Spencer AU, et al. (2007) Intestinal epithelial cell proliferation is dependent on the site of massive small bowel resection. Paediatric Surg Int $\mathbf{2 3}$, 379-390.

32. Torres DM, Tooley KL, Butler RN, et al. (2008) Lyprinol only partially improves indicators of small intestinal integrity in a rat model of 5-fluorouracil-induced mucositis. Cancer Biol Ther 7, 295-302. 